\title{
Outcomes of liver-kidney transplantation in patients with primary hyperoxaluria: an analysis of the scientific registry of transplant recipients database
}

Jie Xiang ${ }^{1 \dagger}$, Zheng Chen ${ }^{1 \dagger}$, Fangshen $\mathrm{Xu}^{1,2}$, Shengmin Mei ${ }^{1}$, Zhiwei $\mathrm{Li}^{1}$, Jie Zhou', Yinlei Dong ${ }^{1}$, Yangjun Gu${ }^{1}$, Zhichao Huang ${ }^{1}$ and Zhenhua $\mathrm{Hu}^{1,2,3^{*}}$

\begin{abstract}
Background: Primary hyperoxaluria (PH) is an inherited disease lacking of hepatic oxalic acid metabolic enzymes which could lead to irreverisible renal damage. Currently, liver-kidney transplantation is a curative but highly invasive therapy used to treat patients with PH. However, limited studies have focused on combined liver-kidney transplantation (CLKT) and sequential liver and kidney transplantation (SLKT) in patients with PH.

Methods: The present study included 201 patients with PH who received both liver and kidney transplants and who were listed on the Scientific Registry of Transplant Recipients from 1987 to 2018 . According to the liver-kidney transplant procedure, patients were separated into a CLKT group and a SLKT group. Patient demographics and transplant outcomes were assessed in each group.

(Continued on next page)
\end{abstract}

\footnotetext{
* Correspondence: huzhenh@zju.edu.cn

†Jie Xiang and Zheng Chen contributed equally to this work.

'Division of Hepatobiliary and Pancreatic Surgery, Department of Surgery,

First Affiliated Hospital, School of Medicine, Key Laboratory of Combined Multi-Organ Transplantation, Ministry of Public Health Key Laboratory of Organ Transplantation, Zhejiang University, No.79 Qingchun Road, Hangzhou

310003, Zhejiang Province, China

${ }^{2}$ Division of Hepatobiliary and Pancreatic Surgery, Department of Surgery,

Fourth Affiliated Hospital, School of Medicine, Zhejiang University, Yiwu,

Zhejiang, China

Full list of author information is available at the end of the article
}

(C) The Author(s). 2020 Open Access This article is licensed under a Creative Commons Attribution 4.0 International License, which permits use, sharing, adaptation, distribution and reproduction in any medium or format, as long as you give appropriate credit to the original author(s) and the source, provide a link to the Creative Commons licence, and indicate if changes were made. The images or other third party material in this article are included in the article's Creative Commons licence, unless indicated otherwise in a credit line to the material. If material is not included in the article's Creative Commons licence and your intended use is not permitted by statutory regulation or exceeds the permitted use, you will need to obtain permission directly from the copyright holder. To view a copy of this licence, visit http://creativecommons.org/licenses/by/4.0/. The Creative Commons Public Domain Dedication waiver (http://creativecommons.org/publicdomain/zero/1.0/) applies to the data made available in this article, unless otherwise stated in a credit line to the data. 
(Continued from previous page)

Results: Compared with the SLKT group, The CLKT group got a worse pretransplant dialysis condition in both the proportion of patients under pretransplant dialysis $(p=0.048)$ and the duration of the pretransplant dialysis $(p<$ 0.001). The SLKT group got higher human leukocyte antigen mismatch score of kidney donor $(p<0.001)$ and liver donor $(p=0.003)$. The CLKT group utilized higher proportion (98.9\%) of organs from a single deceased donor, while the SLKT group utilized $75.0 \%$ of organs from deceased liver donors and only $35.0 \%$ of organs from deceased kidney donors $(p<0.001)$. Kidney function measured by serum creatinine concentration before liver transplantation $(\mathrm{LT})$ or CLKT was similar ( $p=0.305)$ between groups. Patient survival was not significantly different between the two groups ( $p=0.717)$ and liver $(p=0.685)$ and kidney $(p=0.464)$ graft outcomes were comparable between the two groups.

Conclusions: SLKT seems to be an alternative option with strict condition for CLKT, further exploration about the SLKT is still required.

Keywords: Primary hyperoxaluria, Inherited disease, Liver-kidney transplantation, Combined liver and kidney transplantation, Sequential liver and kidney transplantation

\section{Background}

Primary hyperoxaluria $(\mathrm{PH})$ is a kind of congenital disorder that results from abnormal glyoxylate metabolism owing to deficiency of hepatic enzymes [1]. However, symptoms of $\mathrm{PH}$ are usually observed in the urinary tract, and are related to continuous oxalate deposition and irreversible renal damage resulting in end-stage kidney disease (ESKD). In patients with ESKD, a high level of oxalate production is compounded by a high daily dietary oxalate intake and impaired oxalate elimination, which causes a step-by-step increase in plasmatic oxalate and results in calcium oxalate crystals depositions in extra-renal tissues and organs, such as the skin, bones, and heart [2]. Systemic oxalosis is observed in the later stage of ESKD and can be fatal in patient with $\mathrm{PH}$ [3]. Patients with type $1 \mathrm{PH}$ (PH1), which is induced by loss of hepatic alanineglyoxylate aminotransferase [4] account for the majority of patients with $\mathrm{PH}$ worldwide $[5,6]$. Since simple renal therapies such as dialysis or kidney transplant [1] are not able to solve oxalate accumulation, more effective strategies are under exploration.

Liver-kidney transplantation (LKT) is proposed because it could not only rescue hepatic enzyme deficiency, but it could also replace the dysfunctional kidney, especially in patients with PH1. Combined liver and kidney transplantation (CLKT) is the mainstream transplant strategy for LKT procedures, and global centers have confirmed the feasibility of the procedure with encouraging long-term results, even in pediatric patients $[7,8]$. However, this procedure demands liver and kidney donors, usually a single deceased donor, which is costly and strict owing to organ shortages [9]. Sequential liver and kidney transplantation (SLKT) is an alternative strategy that separates the CLKT procedure into two steps: liver transplant (LT) and kidney transplant (KT). The application of intensive dialysis after LT could help the liver graft to mobilize and eliminate the general oxalate burden before $\mathrm{KT}$, which may improve renal graft survival outcomes $[10,11]$. However, previous studies pointed out the inferior patient survial in SLKT or CLKT compared with kidney transplant in children, and these studies may suggest the the overall high risk of multiorgan transplantation, even in sequential procedure $[8,12]$. There were researchers believed that liver allograft provided immunoprotection for kidney graft only if both organs are transplanted simultaneously [13]. But recently, Kumiko et al. pointed out that the preceding liver allograft could contribute to the immunological protection, which may benefit the long term renal allograft outcome in patients after SLKT from a single donor when compared with kidney transplant alone [9]. However, the details of the SLKT in specific disease condition like $\mathrm{PH}$, such as the donorrecipient match condition, the timings of LT and sequential $\mathrm{KT}$, are still not reach a consensus.

Although different series and registry studies about $\mathrm{PH}$ have been published worldwide, most of them adopted a single-center design. The limited number of patients in these cohorts means that the results are not statistically significant. Little is known about multi-center research into CLKT and SLKT in patients with PH. Calinescu, A. $M$. et al. analyzed all primary pediatric CLKT cases in the Scientific Registry of Transplant Recipients (SRTR) and found that CLKT has similar survival outcomes when compared with isolated LT (including SLKT) and patients with $\mathrm{PH}$ present with worse outcomes than patients without $\mathrm{PH}$ [8]. The details in the group of patients with $\mathrm{PH}$ were not mentioned in this study. In this study, we sought to clarify the differences between CLKT and SLKT in patients with $\mathrm{PH}$ in the SRTR database to involve patients reported to date as much as possible.

\section{Methods}

Data source

This study was based on the SRTR, which includes data from all donors, wait-listed candidates, and transplant 
recipients in the U.S.A., submitted by the members of the Organ Procurement and Transplantation Network (OPTN). The Health Resources and Services Administration (HRSA), the U.S. Department of Health and Human Services, provides oversight into the activities of the OPTN and SRTR contractors. The whole study was reviewed and approved by the ethics committee at Zhejiang University, China (No.2019-1022-1).

\section{Study population}

$\mathrm{PH}$ was defined using specific diagnosis codes (code 4307 in the LT database, code 3013 in the KT database) or indications of $\mathrm{PH}$ specified in the diagnosis text field. After data integration, only patients from 1987 to 2018 underwent CLKT or SLKT (with prior LT) according to the time interval between LT and KT. Patients who had received a multi-visceral transplant or who underwent re-transplantation were excluded from the study.

\section{Study outcomes}

The primary outcomes were post-transplant patient survival, liver graft survival, and kidney graft survival. Patient survival was defined as the time from the initial transplant to patient death or final follow up. Liver graft survival was defined by the time interval between date of LT and liver graft failure date, patient death date, or final follow up date. Kidney graft survival was defined by the time interval from date of KT to kidney graft dysfunction date, patient death date, or final follow up date. Either liver or kidney graft dysfunction was defined by a clear graft failure record or re-transplantation date.

\section{Statistics}

Demographic and clinical characteristics of recipients at initial transplant and liver and kidney donor and graft characteristics were described using median (interquartile range [IQR]) and frequency (percentages), respectively. Continuous and categorical variables of the analyzed groups were compared using the Mann-Whitney U test and chi-squared tests or Fisher's exact test, as appropriate. A two-sided $\alpha$ level of $<0.05$ indicated statistical significance. The Kaplan-Meier method was applied to estimate the probability of patient survival and graft survival, and the log-rank test was used to make between-group comparisons. Statistical analysis was performed using SPSS 22.0 (IBM Corp, Armonk, NY, U.S.A.).

\section{Results}

According to our definition, a total of 201 patients from 1987 to 2018 were included in this study; 181 patients underwent CLKT, while 20 patients underwent SLKT.

The demographic and clinical features of recipients at initial transplantation are outlined in Table 1 . We found that the pretransplant dialysis condition were significantly different between groups including pretransplant dialysis type and dialysis duration. The proportion of patients under dialysis (including hemodialysis and peritoneal dialysis) was higher in the CLKT group compared with the SLKT group $(87.9 \%$ vs. $64.3 \%, p=0.048)$, and the pretransplant dialysis duration was also found longer in the CLKT group than the SLKT group (17.0 months (IQR 7.0, 22.0 ) vs. 4.0 months (IQR $0.0,12.3$ ), $p<0.001$ ). There were no significant differences between the two groups in terms of age $(p=0.183)$, body mass index $(p=0.133)$, gender $(p=0.651)$, race $(p=0.378)$, and serum creatinine concentration $(p=0.312)$. The median waiting time from the listing date to the initial transplant date was 5.0 (IQR 1.5, 9) in the CLKT group compared with 2 (IQR 1.0, 7.5) in the SLKT group $(p=0.094)$.

Donor and graft characteristics are summarized in Table 2. For liver donors, the CLKT group used a higher proportion of deceased donors (98.9\%) when compared with the SLKT group $(75.0 \% ; P<0.001)$. As for LT procedure type, the proportion of patients receiving whole liver graft was higher in the CLKT group compared with the SLKT group $(80.1 \%$ vs. $60.0 \% ; P=0.025)$. Donor age, gender, race, and cause of death were similar between groups. In kidney donors, the donor age was higher in the CLKT group (median age $=20$ years) compared with the SLKT group (median age $=34$ years; $p<0.001$ ). The CLKT group received organs from deceased donors in a greater percentage of cases (98.9\%) compared with the SLKT group $(35.0 \% ; p<0.001)$. Donor gender, race, serum creatinine concentration, and cause of death were similar between groups.

Transplant outcomes are listed in Table 3. For liver outcomes, the incidence of rejection episodes between transplant and discharge was similar (12.9\% in the CLKT group vs. $12.5 \%$ in the SLKT group, $p=0.898$ ). Although not statistically significant $(p=0.371)$, no incidences of liver graft failure were observed in the SLKT group before discharge, while three patients (3.9\%) developed graft failure in the CLKT group. Discharge laboratory values also demonstrated comparable outcomes in alanine aminotransferase, aspartate aminotransferase, alkaline phosphate, and albumin; while serum creatinine concentration was significantly different $(p=0.018)$.

The incidence of rejection in KT between transplant and discharge was $5 \%$ in the CLKT group, while no rejection was observed in the SLKT group $(p=0.505)$. The incidence of graft failure was $8.3 \%$ in the CLKT group compared with $15 \%$ in the SLKT group $(p=0.321)$. The serum creatinine concentration at discharge was slightly lower in the SLKT group, although statistical significance was not reached $(p=0.161)$. The duration of hospitalization after transplantation in the SLKT group (median time $=10$ days; IQR 5,21 ) was much shorter than in the CLKT group (median time $=19$ days, IQR 9, 
Table 1 Recipient characteristics at initial transplantation in CLKT and SLKT groups

\begin{tabular}{|c|c|c|c|}
\hline Recipient characteristics & CLKT $(\boldsymbol{n}=181)$ & $\operatorname{SLKT}(\boldsymbol{n}=20)$ & $\boldsymbol{P}$ value \\
\hline Median age, year (IQR) & $20.0(8.0,31.5)$ & $11.5(1.85,27.3)$ & 0.183 \\
\hline BMI (median, IQR) & $21.5(18.0,26.9)$ & $18.8(16.9,23.1)$ & 0.133 \\
\hline Gender & & & 0.651 \\
\hline Male & 99 (54.7\%) & $12(60.0 \%)$ & \\
\hline Female & $82(45.3 \%)$ & $8(40.0 \%)$ & \\
\hline Race & & & 0.378 \\
\hline White & $135(74.6 \%)$ & 17 (85.0\%) & \\
\hline Black & $12(6.6 \%)$ & $1(5.0 \%)$ & \\
\hline American Indian or Alaskan Native & $2(1.1 \%)$ & $1(5.0 \%)$ & \\
\hline Others & $1(0.6 \%)$ & $0(0.0 \%)$ & \\
\hline Asian & $8(4.4 \%)$ & $0(0.0 \%)$ & \\
\hline Hispanic & $23(12.7 \%)$ & $1(5.0 \%)$ & \\
\hline Serum creatinine (mg/dL, median, IQR) & $5.1(3.4,7.2)$ & $5.6(2.8,10.7)$ & 0.312 \\
\hline Pretransplant dialysis type & & & 0.048 \\
\hline No dialysis & $11(8.3 \%)$ & $3(21.4 \%)$ & \\
\hline Hemodialysis & $100(75.8 \%)$ & $6(42.9 \%)$ & \\
\hline Peritoneal Dialysis & $16(12.1 \%)$ & $3(21.4 \%)$ & \\
\hline Dialysis Status Unknown & $5(3.8 \%)$ & $2(14.3 \%)$ & \\
\hline Pretransplant dialysis duration (median, IQR) & $17.0(7.0,22.0)$ & $4.0(0.0,12.3)$ & $<0.001$ \\
\hline Median waiting time from listing to transplant, months (IQR) & $5.0(1.5,9)$ & $2(1.0,7.5)$ & 0.094 \\
\hline
\end{tabular}

CLKT Combined liver and kidney transplant, SLKT Sequential liver and kidney transplant, IQR Interquartile range, BMI Body mass index, HLA Human leukocyte antigen

34; $p=0.009)$. The median interval between LT and KT was 10.5 months (IQR 6.25, 24.25).

Cumulative 1-year, 5-year, and 10-year patient survival rates were $89.2,77.0$, and $67.0 \%$ in the CLKT group; and $100,84.1$, and $84.1 \%$ in the SLKT, respectively (Fig. $1, p=$ 0.338). Although not statistically significant, the SLKT group presented with better outcomes. The cumulative 5-year liver graft survival rate was $86.8 \%$ in the CLKT group, and $88.2 \%$ in the SLKT group (Fig. 2, $p=0.685$ ). The cumulative 5-year kidney graft survival rate was $78.1 \%$ in the CLKT group and $85.0 \%$ in the SLKT group (Fig. 3, $p=0.464$ ).

\section{Discussion}

LKT is the only curable treatment for $\mathrm{PH}$ because it solves both hepatic enzyme disorder and renal dysfunction, in which the CLKT is a globally implemented LKT procedure with abundant experience. However, SLKT seems to be a much more feasible procedure because it divides the LKT into two steps: LT followed by KT. The application of posttransplant intensive dialysis could help liver graft to be free from damage caused by a high degree of system oxalate deposition. This procedure has succeeded in living donor transplants with encouraging outcomes [14]. Outcomes of LKT in patients with $\mathrm{PH} 1$ have improved over the decades, particularly in CLKT [15]; however, published data investigating SLKT is limited.
Some clinicians have highlighted that SLKT suits patients with long-term renal replace treatment or patients suffering with systemic oxalosis, while CLKT suits patients with a short dialysis period or without systemic oxalosis [14]. Our study demonstrated that the SLKT group had similar preoperative renal function at LT compared with the CLKT group, and the long-term outcomes of patient survival and graft loss were encouraging in the SLKT group when compared with the CLKT group. The cumulative 1-year, 5-year, and 10-year patient survival rates were 89.2, 77.0, 67.0\% in the CLKT group, and 100, 84.1, $84.1 \%$ in the SLKT group. The cumulative 5-year liver graft survival rate was $86.8 \%$ in the CLKT group and $88.2 \%$ in the SLKT group, and the cumulative 5-year kidney graft survival rate was $78.1 \%$ in the CLKT group and $85.0 \%$ in the SLKT group. However, these two groups of patients seemed to be at a different level of pretransplant renal condition demonstrated by the higher proportion of patients under dialysis and longer pretransplant duration (17.0 months in CLKT (IQR 7.0, 22.0) vs. 4.0 months in SLKT (IQR 0.0, 12.3), $p<0.001$ ) in the CLKT group compared with the SLKT group. Then we simply compared pretransplant renal function between 2 groups by serum creatinine because of lacking of GFR data before LT in SLKT groups, however, the serum creatinine showed no significant difference $(p=0.312)$. 
Table 2 Donor and graft characteristics in CLKT and SLKT groups

\begin{tabular}{|c|c|c|c|}
\hline Donor and graft characteristics & $\mathrm{CLKT}(\boldsymbol{n}=181)$ & SLKT $(\boldsymbol{n}=20)$ & $\boldsymbol{P}$ value \\
\hline \multicolumn{4}{|l|}{ Liver } \\
\hline Median age, year (IQR) & $20.0(12.0,30.5)$ & $21.0(10.0,37.5)$ & 0.977 \\
\hline Gender & & & 0.492 \\
\hline Male & $105(58.0 \%)$ & $10(50.0 \%)$ & \\
\hline Female & $76(42.0 \%)$ & $10(50.0 \%)$ & \\
\hline Race & & & 0.087 \\
\hline White & $116(64.1 \%)$ & 19 (95.0\%) & \\
\hline Black & $22(12.2 \%)$ & $1(5.0 \%)$ & \\
\hline American Indian or Alaskan Native & $2(1.1 \%)$ & $0(0.0 \%)$ & \\
\hline Asian & $4(2.2 \%)$ & $0(0.0 \%)$ & \\
\hline Hispanic & $37(20.4 \%)$ & $0(0.0 \%)$ & \\
\hline Serum creatinine (mg/dL, median, IQR) & $0.80(0.60,1.00)$ & $0.71(0.44,0.90)$ & 0.243 \\
\hline HLA mismatch & & & 0.003 \\
\hline 0 & $0(0.0 \%)$ & $1(5.9 \%)$ & \\
\hline 1 & $2(1.3 \%)$ & $1(5.9 \%)$ & \\
\hline 2 & $2(1.3 \%)$ & $2(11.8 \%)$ & \\
\hline 3 & $13(8.2 \%)$ & $2(11.8 \%)$ & \\
\hline 4 & $42(26.6 \%)$ & $4(23.5 \%)$ & \\
\hline 5 & $62(39.2 \%)$ & $5(29.4 \%)$ & \\
\hline 6 & 37 (23.4\%) & $2(11.8 \%)$ & \\
\hline Liver transplant procedure & & & 0.025 \\
\hline Whole liver & 145 (80.1\%) & $12(60.0 \%)$ & \\
\hline Partial liver & $13(7.2 \%)$ & $5(25.0 \%)$ & \\
\hline Split liver & $23(12.7 \%)$ & $3(15 \%)$ & \\
\hline Donor type & & & $<0.001$ \\
\hline Deceased & 179 (98.9\%) & $15(75.0 \%)$ & \\
\hline Living & $2(1.1 \%)$ & $5(25.0 \%)$ & \\
\hline Donor cause of death & & & 0.937 \\
\hline Anoxia & $40(22.3 \%)$ & $3(20.0 \%)$ & \\
\hline Cerebrovascular/stroke & $33(18.4 \%)$ & $3(20.0 \%)$ & \\
\hline Head trauma & $96(53.6 \%)$ & $8(53.3 \%)$ & \\
\hline CNS tumor & $4(2.2 \%)$ & $0(0.0 \%)$ & \\
\hline Other & $6(3.4 \%)$ & $1(6.7 \%)$ & \\
\hline \multicolumn{4}{|l|}{ Kidney } \\
\hline Median age, year (IQR) & $20.0(12.0,31.0)$ & $34.0(28.0,43.8)$ & $<0.001$ \\
\hline Gender & & & 0.287 \\
\hline Male & $77(42.5 \%)$ & $11(55.0 \%)$ & \\
\hline Female & $104(57.5 \%)$ & $9(45.0 \%)$ & \\
\hline Race & & & 0.169 \\
\hline White & $117(64.6 \%)$ & 18 (90.0\%) & \\
\hline Black & $22(12.2 \%)$ & $2(10.0 \%)$ & \\
\hline American Indian or Alaskan Native & $2(1.1 \%)$ & $0(0.0 \%)$ & \\
\hline Asian & $4(2.2 \%)$ & $0(0.0 \%)$ & \\
\hline Hispanic & $36(19.9 \%)$ & $0(0.0 \%)$ & \\
\hline
\end{tabular}


Table 2 Donor and graft characteristics in CLKT and SLKT groups (Continued)

\begin{tabular}{|c|c|c|c|}
\hline Donor and graft characteristics & $\mathrm{CLKT}(\boldsymbol{n}=181)$ & $\operatorname{SLKT}(\boldsymbol{n}=20)$ & $\boldsymbol{P}$ value \\
\hline Serum creatinine (mg/dL, median, IQR) & $0.80(0.60,1.00)$ & $1.00(0.23,1.55)$ & 0.771 \\
\hline HLA mismatch & & & $<0.001$ \\
\hline 0 & $0(0.0 \%)$ & $2(10.5 \%)$ & \\
\hline 1 & $2(1.3 \%)$ & $2(10.5 \%)$ & \\
\hline 2 & $2(1.3 \%)$ & $2(10.5 \%)$ & \\
\hline 3 & $13(8.2 \%)$ & $3(15.8 \%)$ & \\
\hline 4 & $42(26.4 \%)$ & $6(31.6 \%)$ & \\
\hline 5 & $63(39.6 \%)$ & $3(15.8 \%)$ & \\
\hline 6 & $37(23.3 \%)$ & $1(5.3 \%)$ & \\
\hline Kidney transplant procedure & & & 0.698 \\
\hline Left kidney & $110(60.8 \%)$ & $13(65.0 \%)$ & \\
\hline Right kidney & $65(35.9 \%)$ & $7(35.0 \%)$ & \\
\hline En bloc & $6(3.3 \%)$ & $0(0.0 \%)$ & \\
\hline Donor type & & & $<0.001$ \\
\hline Deceased & $179(98.9 \%)$ & $7(35.0 \%)$ & \\
\hline Living & $2(1.1 \%)$ & $13(65.9 \%)$ & \\
\hline Donor cause of death & & & 0.622 \\
\hline Anoxia & $40(22.3 \%)$ & $2(28.6 \%)$ & \\
\hline Cerebrovascular/stroke & $33(18.4 \%)$ & $1(14.3 \%)$ & \\
\hline Head trauma & $96(53.6 \%)$ & $3(42.9 \%)$ & \\
\hline CNS tumor & $4(2.2 \%)$ & $0(0.0 \%)$ & \\
\hline Other & $6(3.4 \%)$ & $1(14.3 \%)$ & \\
\hline
\end{tabular}

CLKT Combined liver and kidney transplant, SLKT Sequential liver and kidney transplant, IQR Interquartile range, BMI Body mass index, CNS Central nervous system

Donor condition should also take into consideration. Most CLKT procedures included in the present study utilized organs from single deceased donors. For SLKT, one of the organs is often procured from a living donor and the other from a deceased donor [16-18]. We also demonstrate this organ allocation preference in our patient's demographic statistics analysis: the CLKT group utilized 98.9\% of organs from a single deceased donor, while the SLKT group utilized $75 \%$ of livers from deceased donors and only $35 \%$ of kidneys from deceased donors.

Research suggests that liver grafts have a protective effect on kidney grafts when procured from the same donor and when transplanted simultaneously, which translates to a low rate of acute rejection and improved kidney graft survival when compared with isolated KT or LT $[19,20]$. human leukocyte antigen (HLA) are one the suggested mechanisms behind the immunoprotective phenomenon $[21,22]$. HLA antigens produced by the transplanted liver allograft could neutralize circulating alloantibodies. Soluble HLA antigens delivered by the liver allograft could inhibit natural killer and cytotoxic $\mathrm{T}$ cells. Kupfer cells and biliary epithelial cells from the liver allograft also demonstrate an ability to clear circulating antigens [23, 24]. Our study may support this hypothesis through the observation that the
SLKT group acquired significantly lower mismatch scores when compared with the CLKT group both in liver grafts $(p=0.003)$ and renal grafts $(p<0.001)$ but reached a comparable long-term outcome either in patient survival or graft loss.

Even though the deficiency in specific hepatic enzymes could be solved by liver allograft, renal allograft may accumulate oxalate deposits while mobilizing and eliminating systemic oxalate accumulations $[18,25]$. Therefore, SLKT seems to be more reasonable than CLKT for patients with severe plasma oxalate accumulation because it decrease the plasma oxalate level below the saturation level by LT and intensified dialysis protocol to create a better kidney graft survival condition before KT. Studies have reported that patients undergoing SLKT who underwent an early LT could acquire decreased perioperative morbidity and therefore more choices for kidney allocation (either a deceased or a living donor) compared with those only undergoing dialysis [26]. However, the optimal interval between LT and KT is unknown. In 2017 a United Network for Organ Sharing (UNOS) allocation policy for CLKT was implemented, which defines the medical eligibility criteria for CLKT and provides a safety net by assigning priority for renal allograft allocation to LT recipients with ESKD 
Table 3 Transplant outcomes in CLKT and SLKT groups

\begin{tabular}{|c|c|c|c|}
\hline & $\operatorname{CLKT}(\boldsymbol{n}=181)$ & $\operatorname{SLKT}(\boldsymbol{n}=20)$ & $\boldsymbol{P}$ value \\
\hline \multicolumn{4}{|l|}{ Liver } \\
\hline Rejection episode & $15(12.9 \%)$ & $1(12.5 \%)$ & 0.898 \\
\hline Graft failure & $7(3.9 \%)$ & $0(0.0 \%)$ & 0.371 \\
\hline \multicolumn{4}{|l|}{ Cause of graft failure } \\
\hline Primary graft failure & $2(33.3 \%)$ & & \\
\hline Vascular thrombosis & $2(33.3 \%)$ & & \\
\hline Acute rejection & $1(16.7 \%)$ & & \\
\hline Other & $1(16.7 \%)$ & & \\
\hline \multicolumn{4}{|l|}{ Recipient discharge labs (Median, IQR) } \\
\hline AST $(U / L)$ & $44.0(22.3,61.8)$ & $35.5(26.0,58.3)$ & 0.912 \\
\hline $\operatorname{ALT}(U / L)$ & $44.5(21 ., 97.5)$ & $38(20,88.5)$ & 0.58 \\
\hline Alkaline phosphate $(U / L)$ & $250.5(191,501.8)$ & $441.5(254.0,891.3)$ & 0.083 \\
\hline Albumin (g/dL) & $3.0(2.43,3.38)$ & $2.9(2.0,3.6)$ & 0.626 \\
\hline Serum creatinine (mg/dL) & $1.4(0.7,2.4)$ & $4.4(3.7,6.25)$ & 0.018 \\
\hline Median days from transplant until discharge (IQR) & $19(9,34)$ & $26(14,33)$ & 0.238 \\
\hline \multicolumn{4}{|l|}{ Kidney } \\
\hline Rejection episode & $5(4.3 \%)$ & $0(0.0 \%)$ & 0.505 \\
\hline Graft failure & $15(8.3 \%)$ & $3(15.0 \%)$ & 0.321 \\
\hline \multicolumn{4}{|l|}{ Cause of graft failure } \\
\hline Acute rejection & $0(0.0 \%)$ & $1(33.3 \%)$ & \\
\hline Primary graft failure & $3(20.0 \%)$ & $1(33.3 \%)$ & \\
\hline Graft thrombosis & $2(13.3 \%)$ & $1(33.3 \%)$ & \\
\hline Infection & $1(6.7 \%)$ & $0(0.0 \%)$ & \\
\hline Urological Complications & $1(6.7 \%)$ & $0(0.0 \%)$ & \\
\hline Recurrent diseases & $1(6.7 \%)$ & $0(0.0 \%)$ & \\
\hline Primary non-function & $2(13.3 \%)$ & $0(0.0 \%)$ & \\
\hline Unknown & $5(33.3 \%)$ & $0(0.0 \%)$ & \\
\hline Serum creatinine at discharge $(\mathrm{mg} / \mathrm{dL}$, median, IQR) & $1.3(0.7,2.3)$ & $0.9(0.45,1.4)$ & 0.161 \\
\hline Median number of days from transplant until discharge (IQR) & $19(9,34)$ & $10(5,21)$ & 0.009 \\
\hline Median interval between LT and KT (month, IQR) & & $10.5(6.25,24.25)$ & \\
\hline
\end{tabular}

CLKT Combined liver and kidney transplant, SLKT Sequential liver and kidney transplant, IQR Interquartile range, BMI Body mass index, CNS Central nervous system, $L T$ Liver transplantation, KT Kidney transplantation, AST Aspartate aminotransferase, ALT Alanine aminotransferase

within 1 year of LT [27, 28]. In the present study, the median interval between KT and LT was 10.5 months (IQR $6.25,24.25)$ and the long-term outcome somewhat proved the validity of these allocation policies.

The present study is limited by its retrospective design. First, the diagnosis of $\mathrm{PH}$ is obscure owing to lack of genetic diagnosis data, so the subtype of PH could not be analyzed. Second, the details of patient status, such as oxalate concentration and glomerular filtration rate were not mentioned; we could only simply assess renal function using the recipient serum creatinine concentration and pretransplant dialysis condition. Third, our result may have overestimated the outcome of SLKT because the cases we retrieved from the database were with definite LKT records, making the cases who intended to receive SLKT but failed by the failure of LT were excluded. Furthermore, the sample size was relatively small; a few cases of post-transplant death and graft loss were recorded, making it hard to identify risk factors and perform multivariate assessment.

In summary, $\mathrm{PH}$ is a rare inherited disease that is associated with substantial metabolic disorders and high mortality if it is not treated effectively, and LKT is critical for the majority of patients with $\mathrm{PH}$. Our results may suggest that SLKT could be a viable alternative treatment to CLKT in specific patients with limited conditions including early LT procedure and appropriate donor resource. Based on this suggestion, further 


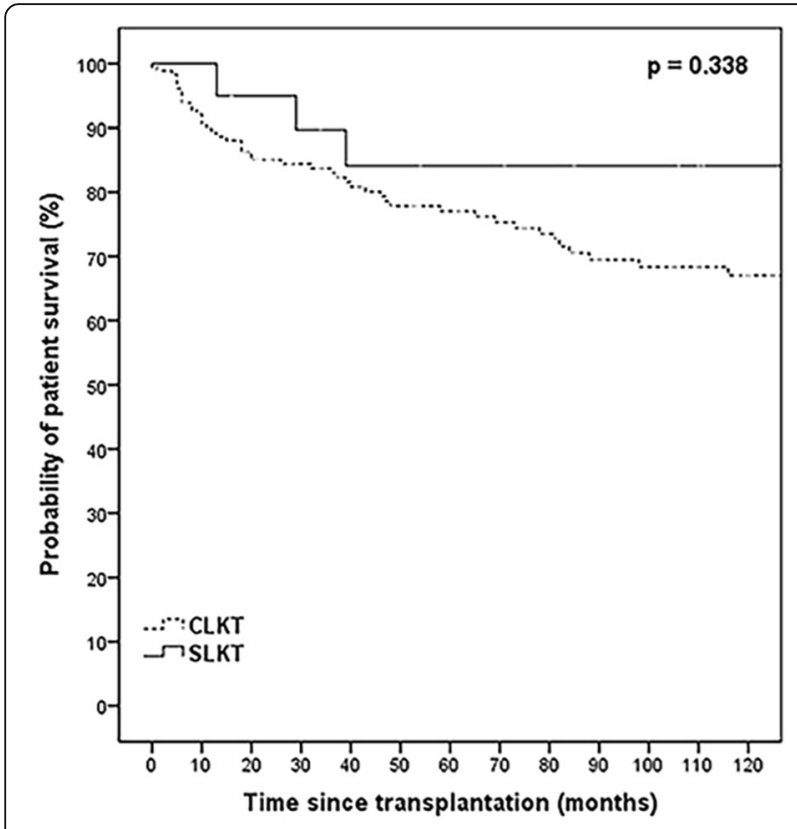

Fig. 1 Kaplan-Meier curves for patient survival in the CLKT and SLKT groups. Recipients in CLKT had nonsignificant survival outcome compared with recipients in SLKT. Continuous line depicts recipients underwent SLKT and dashed line depicts recipients underwent CLKT

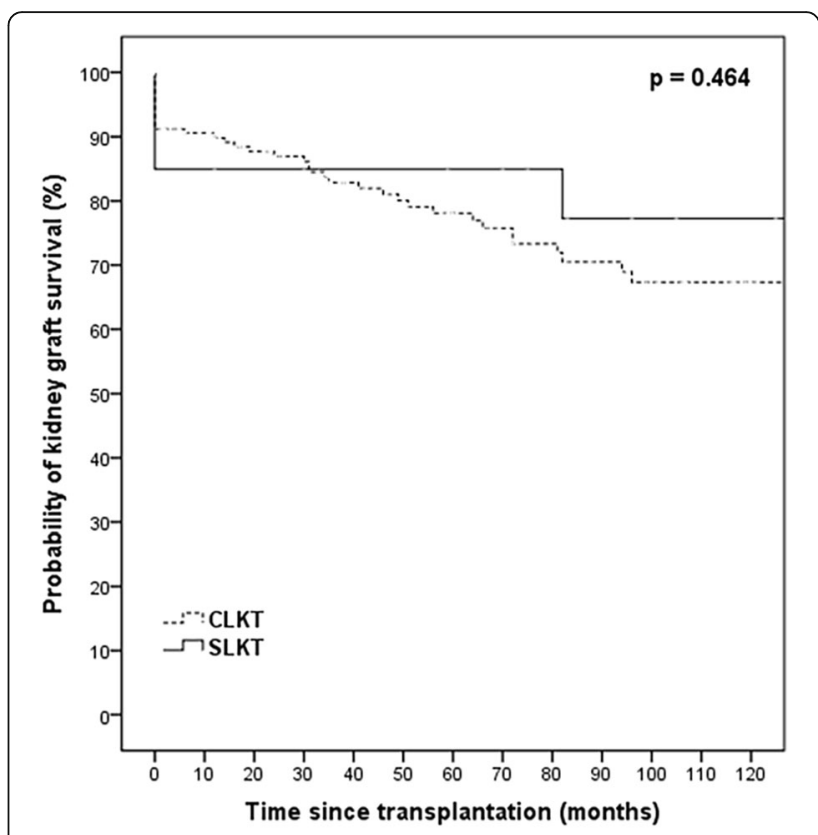

Fig. 3 Kaplan-Meier curves for kidney graft survival in the CLKT and SLKT groups. Recipients of kidney graft in CLKT had comparable graft survival compared with recipients in SLKT. Continuous line depicts recipients underwent SLKT and dashed line depicts recipients underwent CLKT

exploration about the SLKT such as pretransplant condition, donor allocation and interval between the transplantations is still required.

\section{Conclusions}

In conclusion, our study demonstrated that the SLKT seems to be an alternative option with strict condition for CLKT, further exploration about the SLKT is still required.

\section{Abbreviations}

CLKT: Combined liver and kidney transplant; ESKD: End-stage kidney disease:" HLA: Human leukocyte antigen; KT: Kidney transplant; LKT: Liver-kidney transplant; LT: Liver transplant; PH: Primary hyperoxaluria; PH1: Primary hyperoxaluria type 1; SLKT: Sequential liver and kidney transplant

\section{Acknowledgements}

The data reported here have been supplied by the Hennepin Healthcare Research Institute (HHRI) as the contractor for the Scientific Registry of Transplant Recipients (SRTR). The interpretation and reporting of these data are the responsibility of the authors and should in no way be seen as an official policy of or interpretation by the SRTR or the U.S. Government.

\section{Authors' contributions}

J.X and Z. C contributed equally to this paper as first author. Y.L.D, Y.J.G and Z.C.H retrieved the associated data from the SRTR database. J. X and Z. C performed the statistical analysis. Z. C wrote the first draft of the manuscript. F.S.X, Z.W.L, S.M.M and J. Z contributed intellectual content. All authors contributed to critical revisions to the manuscript. Z.H.H is the corresponding author. All authors approved the final manuscript.

Fig. 2 Kaplan-Meier curves for liver graft survival in the CLKT and SLKT groups. Recipients of liver graft in CLKT had comparable graft survival compared with recipients in SLKT. Continuous line depicts recipients underwent SLKT and dashed line depicts recipients underwent CLKT

\section{Funding}

This work was supported by the Public Welfare Technology Application Research Plan of Zhejiang Province, China (No. 2017C33025), National Natural 
Science Foundation of China (No. 81770645), National Health and Family Planning Commission of China (No. 2018260134), Key Research \& Development Program of Zhejiang Province (No. 2020C03057), Medical and health platform project of Zhejiang Province (No. 2020375961).

\section{Availability of data and materials}

The data that support the findings of this study are available from the Scientific Registry of Transplant Recipients database, but restrictions apply to the availability of these data, which were used under license for the current study, and so are not publicly available. Data are however available from the authors upon reasonable request and with permission of the Scientific Registry of Transplant Recipients database.

\section{Ethics approval and consent to participate}

With Data Release Agreement with the Scientific Registry of Transplant Recipients database, all data in this study were released from SRTR. And no additional patient consent was required for the measurements in the data analysis.

\section{Consent for publication}

Not applicable.

\section{Competing interests}

The authors declare that they have no competing interests.

\section{Author details}

${ }^{1}$ Division of Hepatobiliary and Pancreatic Surgery, Department of Surgery, First Affiliated Hospital, School of Medicine, Key Laboratory of Combined Multi-Organ Transplantation, Ministry of Public Health Key Laboratory of Organ Transplantation, Zhejiang University, No.79 Qingchun Road, Hangzhou 310003, Zhejiang Province, China. ${ }^{2}$ Division of Hepatobiliary and Pancreatic Surgery, Department of Surgery, Fourth Affiliated Hospital, School of Medicine, Zhejiang University, Yiwu, Zhejiang, China. ${ }^{3}$ Division of Hepatobiliary and Pancreatic Surgery, Yiwu Central Hospital, Yiwu, Zhejiang, China.

Received: 3 May 2020 Accepted: 17 June 2020

Published online: 03 July 2020

\section{References}

1. Cochat P, Rumsby G. Primary hyperoxaluria. N Engl J Med. 2013;369(7):649-58.

2. Dindo M, Conter C, Oppici E, Ceccarelli V, Marinucci L, Cellini B. Molecular basis of primary hyperoxaluria: clues to innovative treatments. Urolithiasis. 2019;47(1):67-78.

3. Hoppe B. An update on primary hyperoxaluria. Nat Rev Nephrol. 2012;8(8): 467-75.

4. Danpure CJ, Lumb MJ, Birdsey GM, Zhang X. Alanine:glyoxylate aminotransferase peroxisome-to-mitochondrion mistargeting in human hereditary kidney stone disease. Biochim Biophys Acta. 2003;1647(1):70-5.

5. Cochat $P$, Liutkus A, Fargue $S$, Basmaison $O$, Ranchin B, Rolland MO. Primary hyperoxaluria type 1: still challenging! Pediatr Nephrol. 2006;21(8):1075-81.

6. Lieske JC, Monico CG, Holmes WS, Bergstralh EJ, Slezak JM, Rohlinger AL, Olson JB, Milliner DS. International registry for primary Hyperoxaluria. Am J Nephrol. 2005;25(3):290-6.

7. Bacchetta J, Mekahli D, Rivet C, Demede D, Leclerc AL. Pediatric combined liver-kidney transplantation: a 2015 update. Curr Opin Organ Transpl. 2015; 20(5):543-9.

8. Calinescu AM, Wildhaber BE, Poncet A, Toso C, McLin VA. Outcomes of combined liver-kidney transplantation in children: analysis of the scientific registry of transplant recipients. Am J Transplant. 2014;14(12):2861-8.

9. Kitajima K, Ogawa Y, Miki K, Kai K, Sannomiya A, Iwadoh K, Murakami T, Koyama I, Nakajima I, Fuchinoue S. Longterm renal allograft survival after sequential liver-kidney transplantation from a single living donor. Liver Transpl. 2017;23(3):315-23.

10. Sasaki K, Sakamoto S, Uchida H, Shigeta T, Matsunami M, Kanazawa H, Fukuda A, Nakazawa A, Sato M, Ito S, Horikawa R, Yokoi T, Azuma N, Kasahara M. Two-step transplantation for primary hyperoxaluria: a winning strategy to prevent progression of systemic oxalosis in early onset renal insufficiency cases. Pediatr Transplant. 2015;19(1):E1-6.

11. Mor E, Nesher E, Ben-Ari Z, Weissman I, Shaharabani E, Eizner S, Solomonov E, Rahamimov R, Braun M. Sequential liver and kidney transplantation from a single living donor in two young adults with primary hyperoxaluria type 1. Liver Transpl. 2013;19(6):646-8.

12. Hamdani G, Zhang B, Liu C, Goebel J, Zhang Y, Nehus E. Outcomes of pediatric kidney transplantation in recipients of a previous non-renal solid organ transplant. Am J Transplant. 2017;17(7):1928-34.

13. Simpson N, Cho YW, Cicciarelli JC, Selby RR, Fong TL. Comparison of renal allograft outcomes in combined liver-kidney transplantation versus subsequent kidney transplantation in liver transplant recipients: analysis of UNOS database. Transplantation. 2006:82(10):1298-303.

14. Ozer A, Aktas H, Bulum B, Emiroglu R. The experience of combined and sequential liver and kidney transplantation from a single living donor in patients with primary hyperoxaluria type 1. Pediatr Transplant. 2019;23(4):e13406.

15. Croome KP, Mao S, Yang L, Pungpoapong S, Wadei HM, Taner CB. Improved National Results with Simultaneous Liver and Kidney Transplantation using Donation after Cardiac Death Donors. Liver Transplant : official publication of the American Association for the Study of Liver Diseases and the International Liver Transplantation Society. 2019. https://doi.org/10.1002/lt.25653.

16. Harps E, Brinkert F, Ganschow R, Briem-Richter A, van Husen M, Schmidtke S, Herden U, Nashan B, Fischer L, Kemper MJ. Immediate postoperative intensive care treatment of pediatric combined liver-kidney transplantation: outcome and prognostic factors. Transplantation. 2011;91(10):1127-31.

17. Cochat P, Fargue S, Harambat J. Primary hyperoxaluria type 1: strategy for organ transplantation. Curr Opin Organ Transpl. 2010;15(5):590-3.

18. Illies F, Bonzel KE, Wingen AM, Latta K, Hoyer PF. Clearance and removal of oxalate in children on intensified dialysis for primary hyperoxaluria type 1. Kidney Int. 2006;70(9):1642-8.

19. Opelz G, Margreiter R, Döhler B. Prolongation of long-term kidney graft survival by a simultaneous liver transplant: the liver does it, and the heart does it too. Transplantation. 2002;74(10):1390-71.

20. Rana A, Robles S, Russo MJ, Halazun KJ, Woodland DC, Witkowski P, Ratner $L E$, Hardy MA. The combined organ effect: protection against rejection? Ann Surg. 2008;248(5):871-9.

21. Zino E, Frumento G, Marktel S, Sormani MP, Ficara F, Di Terlizzi S, Parodi AM, Sergeant R, Martinetti M, Bontadini A, Bonifazi F, Lisini D, Mazzi B, Rossini S, Senvida P, Ciceri F, Bonini C, Lanino E, Bandini G, Locatelli F, Apperley J, Bacigalupo A, Ferrara GB, Bordignon C, Fleischhauer K. A T-cell epitope encoded by a subset of HLA-DPB1 alleles determines nonpermissive mismatches for hematologic stem cell transplantation. Blood. 2004;103(4):1417-24.

22. Petersdorf EW, Hansen JA, Martin PJ, Woolfrey A, Malkki M, Gooley T, Storer B, Mickelson E, Smith A, Anasetti C. Major-histocompatibility-complex class I alleles and antigens in hematopoietic-cell transplantation. N Engl J Med. 2001;345(25):1794-U1794.

23. Créput C, Durrbach A, Menier C, Guettier C, Samuel D, Dausset J, Charpentier B, Carosella ED, Rouas-Freiss N. Human leukocyte antigen-G (HLA-G) expression in biliary epithelial cells is associated with allograft acceptance in liver-kidney transplantation. J Hepatol. 2003;39(4):587-94.

24. Knechtle SJ, Kwun J. Unique aspects of rejection and tolerance in liver transplantation. Semin Liver Dis. 2009;29(1):91-101.

25. Bollée $G$, Cochat $P$, Daudon M. Recurrence of crystalline nephropathy after kidney transplantation in APRT deficiency and primary Hyperoxaluria. Can J Kidney Health Dis. 2015;2:69.

26. Gonwa TA, McBride MA, Mai ML, Wadei HM. Kidney transplantation after previous liver transplantation: analysis of the organ procurement transplant network database. Transplantation. 2011;92(1):31-5.

27. Formica RN, Aeder M, Boyle G, Kucheryavaya A, Stewart D, Hirose R, Mulligan D. Simultaneous liver-kidney allocation policy: a proposal to optimize appropriate utilization of scarce resources. Am J Transplant Off J Am Soc Transplant Am Soc Transplant Surg. 2016;16(3):758-66.

28. Asch WS, Bia MJ. New organ allocation system for combined liver-kidney transplants and the availability of kidneys for transplant to patients with stage 4-5 CKD. Clin J Am Soc Nephrol. 2017;12(5):848-52.

\section{Publisher's Note}

Springer Nature remains neutral with regard to jurisdictional claims in published maps and institutional affiliations. 